\title{
Agricultural harvesting using integrated robot system
}

\author{
Vikram Raja, Bindu Bhaskaran, Koushik Karan Geetha Nagaraj, Jai Gowtham Sampathkumar, \\ Shri Ram Senthilkumar \\ Department of Robotics and Automation Engineering, PSG College of Engineering, Coimbatore, India
}

\begin{tabular}{|c|c|}
\hline Article Info & ABSTRACT \\
\hline Article history: & In today's competitive world, robot designs are developed to simplify and \\
\hline Received Jun 3, 2021 & improve quality wherever necessary. The rise in technology and \\
\hline Revised Oct 25, 2021 & sector. The agricultural sector's solution for harvesting fruits and vegetables \\
\hline Accepted Nov 30, 2021 & $\begin{array}{l}\text { is manual labor and a few other agro bots that are expensive and have } \\
\text { various limitations when it comes to harvesting. Although robots present }\end{array}$ \\
\hline Keywords: & $\begin{array}{l}\text { may achieve harvesting, the aftordability of such designs may not be } \\
\text { possible by small and medium-scale producers. The integrated robot system }\end{array}$ \\
\hline Affordability & is designed to solve this problem, and when compared with the existing \\
\hline Agricultural industry & manual methods, this seems to be the most cost-effective, efficient, and \\
\hline Harvesting robot & $\begin{array}{l}\text { viable solution. The robot uses deep learning lor image detection, and the } \\
\text { object is acquired using robotic manipulators. The robot usesian and }\end{array}$ \\
\hline Small-medium scale industry & articulated configuration to perform the picking action. In the end, the robot \\
\hline Vision system & $\begin{array}{l}\text { is operated where carrots and cantaloupes were harvested. The data of the } \\
\text { harvested crops are used to arrive at the conclusion of the robot's accuracy. }\end{array}$ \\
\hline
\end{tabular}

This is an open access article under the CC BY-SA license.

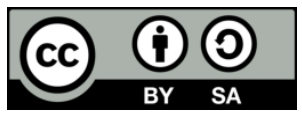

\section{Corresponding Author:}

Vikram Raja

Department of Robotics and Automation Engineering, PSG College of Technology

168 Avinashi Rd, Peelamedu, Coimbatore, Tamil Nadu 641004, India

Email: vikramraja.ur@gmail.com

\section{INTRODUCTION}

The diverse climate and soil of India ensure the obtainability of all different types of fresh fruits and vegetables. India ranks in the production of fruits and vegetables second behind China. During 2015-2016 the National Horticulture database issued that India produced 169.1 million metric tonnes of vegetables and 90.2 million metric tonnes of fruits. The fruits were cultivated at 6.3 million hectares, while the cultivation of fruits stood at 10.1 million hectares. When it comes to ginger and okra, India is the largest producer of vegetables and ranks second in the production of potatoes, brinjal, cabbage, cauliflowers, onions, and other vegetables. In fruits, India ranks first in the production of bananas $(25.7 \%)$, mangoes (40.4\%), and papayas (43.6\%). Export has tremendous opportunities when it comes to the production of fruits and vegetables [1]. During 2019-20, India exported fruits and vegetables which is worth Rs.9,182.88 crores/1,277.38 USD Millions which consisted of and vegetables worth Rs.4,350.13 crores/608.48 USD Millions and fruits worth Rs.4,832.81 crores/668.75 USD Millions. Bangladesh, Oman, Qatar, Nepal, Malaysia, UAE, Netherland, the UK, and Sri Lanka, are the major areas where Indian fruits and vegetables are exported. The production rate of horticulture is increasing at an exponential rate. The input cost and land used are less in horticulture, and this factor is of great benefit. The fruits and vegetables provide a high nutritional value, and usage is higher among the urban population than rural population. The process involved in horticulture, such as harvesting, is a slow, unskilled, tedious, and repetitive job that can be automated. If this process is automated, farmers can work on improving their production yields which will further help the entire society. 


\section{RESEARCH METHOD}

\subsection{Literature review}

Robotics in agriculture was first developed in the 1920s. The first step towards agricultural robotics was the mechanical harvesters. These use a simple and onerous mechanism to gather the harvest. The first mechanical harvest was used to gather tomatoes, which was patented in 1960. Further on, various harvesting systems came into play that has revolutionized this world. In terms of ground-level harvesting, the SW 6010 is the first autonomous robot which is available that can collect strawberries. The other system is called soft harvesting, where delicate suction cups are used to collect the fruits such as pineapples, apples [2], [3]. These robotic arms can also be combined hand to hand to provide better accuracy while gathering. MetoMotion's robotic system is described as 'a multipurpose robotic intensive system for labor-intensive tasks in greenhouses which is to harvest tomatoes [4]. Vinken built is a robot that can see underground. It emits an electric signal which identifies the vegetables [5]. A tomato harvesting system in the form of a mobile robot is capable of harvesting with manual navigation [6]. Various systems exist that harvest strawberries on a semi-automation basis and chili pepper harvesting using the robotic arm [7]-[9]. All these systems are some of the ideas that are present in the market.

The solutions in the market are extravagantly expensive and cannot be afforded by small-medium scale farmers. The labor assigned to take care of the farm must be skilled highly. The consumption of electricity increases as the robots run through electricity. The present harvesting robot requires manual control, but that is not a viable option as the task is to automate the harvesting task. If any technical error occurs, the entire harvesting comes to a halt until the system is fixed. This creates a delayed harvest. A completely automated farm is not a practical possibility as of now.

\subsection{Proposed solution}

The current solutions in the market either involve a mobile harvesting system or robotic arm harvesting, not a combination of both. No model has been executed with the concept of a Cartesian and an articulated robot. The design developed aims to make this system affordable at all levels of farming, and this can be operated at great ease [10]. No manual intervention is necessary for this system as this harvesting robot scans the entire area and yields the output. The accuracy of the yield is of the standard level. The products that can be harvested by this robot design includes cantaloupe, carrot, radish, saffron, pineapple, chili paprika, and all underground vegetable. The first step, as shown in Figure 1, involves the user starting the process through the display unit. As soon as the command is given, it sends the instruction to the cloud server, which communicates to the robot station to begin the process [11]. The robots then, from the docked station, travels to the initial point in the farm to start the harvesting. Once the harvesting is completed, the collected harvest is then delivered to the packing station by the robot. Then the robot travels to the charging point near the robot station and goes to the initial position, and awaits further instruction. A detailed outline of the system is shown in Figure 2.

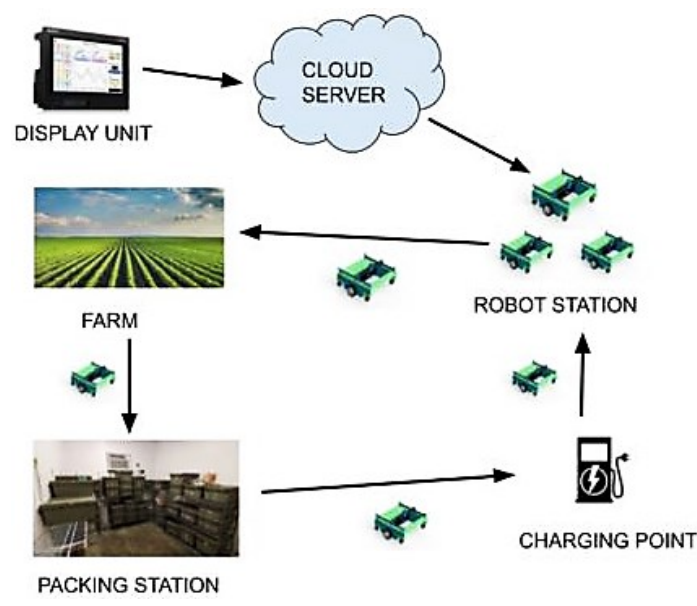

Figure 1. Process of the system

\subsection{Vision system}

The vision system software for the detection of the fruits and vegetables for harvesting uses the YOLO-V3 (you only look once) object detection technique to identify and acquire the location of the fruits. YOLO-V3 uses a Darknet-53 feature extractor which has 53 convolutional neural network layers and skips 
connections inspired by ResNet [12]. It also has a feature pyramid network that allows the YOLO-V3 to learn and detect objects of different sizes. The output of the feature pyramid network is known as a grid, and it has three scales of grids. In each of these grids, three anchor boxes with the same centroid of different sizes are placed on them. These anchor boxes predict the class id and the location of the object. Thus, the bounding boxes are applied to the image [13]. The YOLO-V3 object detection was chosen among many techniques from Figure 3 , which is plotted between common objects in context (COCO) average precision and inference time. It is clear that the YOLO-V3 has the average precision and speed when compared with other methods [14]. The main advantage of using a convolution neural network is that it can even detect a crop if it is covered with leaves [15], [16].

The YOLO-V3 network was trained using Google Colab [17], as it has a powerful graphics processing unit and more compute unified device architecture (CUDA) cores to reduce the overall training time [18]. It took around 5 to 6 hours for 2,000 iterations using 1,000 images of the required crop, which is to be detected 19], [20]. The iterations were performed for carrots and cantaloupe. The camera is 5-megapixel and is equipped with infrared lights. This is useful for the operation of the robot even during night time which leads to greater efficiency in the output. The graph plotted between loss and the no of iterations. After 2,000 iterations, the loss came down almost near to zero. The basic principle is that more loss leads to less detection and less loss leads to an accurate detection.

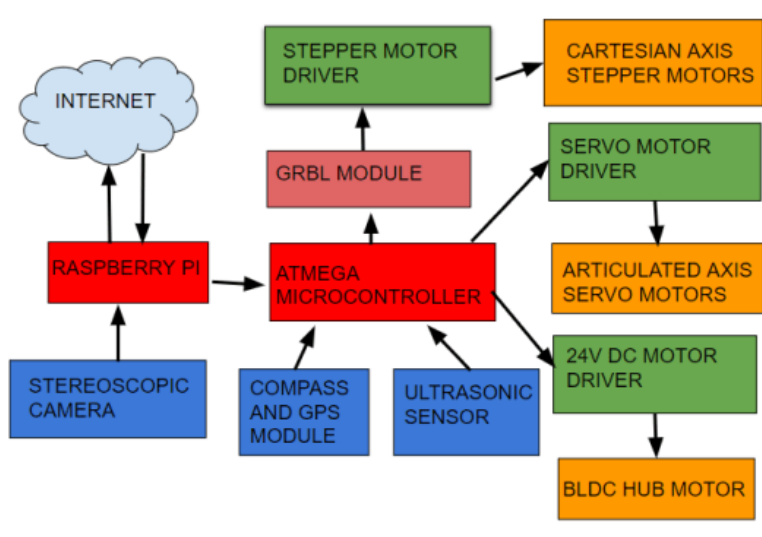

Figure 2. Outline of the system

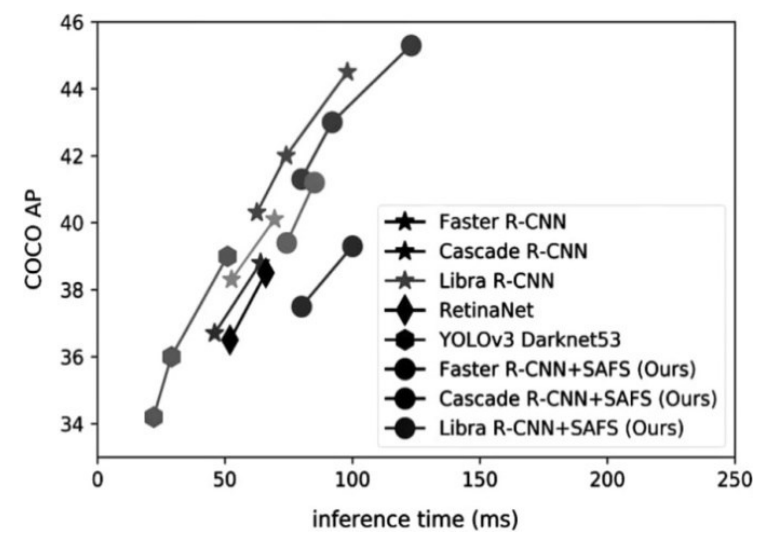

Figure 3. Performance comparison graph

\subsection{Cartesian axis movement}

After locating the crop, the open source computer vision library (OpenCV) software sends the Cartesian coordinates to the universal G Code sender software. Now, this software converts these coordinates into G Codes. Then it is sent to the ATmega microcontroller through serial data transfer. The microcontroller acquires these $\mathrm{G}$ Codes and converts them to pulses for driving the stepper motor and to move the Cartesian robot to the specified location. After this process, the articulated robotic arm which is attached to the Cartesian robot harvests the crop.

\subsection{Navigation}

The localization of the robot is done through global positioning system (GPS) and navigation through waypoints. As it is an outdoor application, light detection and ranging (LiDAR) won't be suitable for this robot [21]. In this case, the QGroundControl software is for creating waypoints and navigating the automated guided vehicle. The concept of precision farming is used to improve accuracy [22], [23]. First, the boundaries of the farm are marked through the QGroundControl software, and then the waypoints are generated for path planning, as shown in Figure 4 [24], [25]. Once the path is planned, the entire area is covered by the robot to obtain all the grown harvest. The software ensures that only fully grown fruits and vegetables are harvested.

\subsection{Modules}

The design of the harvesting bot mainly involves four modules: 1) Automated guided vehicle $(\mathrm{AGV}), 2$ ) Cartesian axis, 3) Articulated axis, and 4) Sensors and navigation. The computer-aided design of the harvesting robot is shown in Figure 5.

The AGV consists of four $150 \mathrm{~mm}$ rubber gripped castor wheels for better friction and traction to the ground. The AGV linear movements are actuated by two $300 \mathrm{~mm}$ wheels with the 24-voltage brushless 
direct current hub motor, and the shaft of the wheels is supported through ball bearings. Two lithium-ion batteries, which have a 24-voltage and current capacity of $10 \mathrm{Ah}$, are connected in parallel so that they can be used as the main power supply. The base frame is crafted through wood as the robot is a prototype and the wooden planks are easily affordable [26].

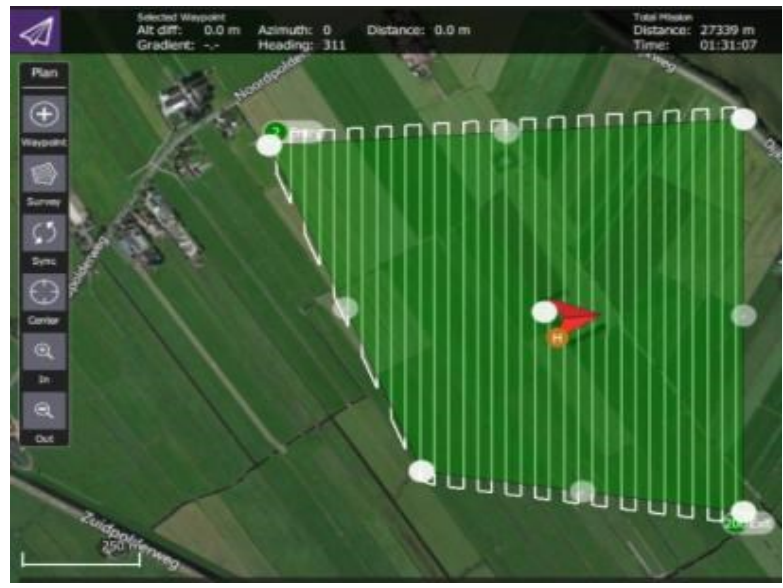

Figure 4. Path planning

The Cartesian axis consists of 4 aluminium V-Slot channels of size $40 \mathrm{~mm}$ length, $20 \mathrm{~mm}$ width, and $1100 \mathrm{~mm}$ height are used as guideways for the V-Wheels [27]. The V-Wheels are attached to the 3D printed components, which act as an end support carrier for the V-Slot channels. The Cartesian axis also includes three lead screws for each X, Y, and Z-axis, which act as linear actuators and are coupled with the Nema 17 stepper motor using a flexible coupler. The flexible couple gives an advantage as a vibration damper. The ends of the lead screws are supported through $8 \mathrm{~mm}$ bore diameter ball bearings. However, as the Z-axis carrier uses linear rods, neglecting the need for V-Slot channels so that linear bearings are used here to support the linear rods. The Articulated robot is a 3D printed six servo motors controlled robotic arm that uses Adafruit servo motor drivers for controlling the servo motors simultaneously. The filament used in 3D printing is polylactic acid plastic (PLA) [28]. The robotic arm is designed through Solid works 3D modeling software, and Inverse kinematics is done through Denavit and Hartenberg parameters [29], [30]. The gripper designed is by considering the object size and by testing in various simulation platforms [31]-[33]. Motor sizing is the crucial part while designing the robotic arm as it determines the torque requirements, and it is done through oriental motor software. Ultrasonic sensors of 3 numbers are used here to avoid any obstacles while the automated guided vehicle is in motion. A stereoscopic image sensor is used here as an input to the image detection and classification software and also to find the depth of the image so that the robotic arm can reach the exact height of the crop and acquire it. For outdoor applications, as the robot is not bounded by any walls, the GPS waypoint navigation is used. The GPS waypoints navigations require inputs such as latitude, longitude, and the direction of the robot so that the global positioning system and compass modules are used here to localize the robot. All these modules are combined in order to give the end result of the final harvesting robot, as shown in Figure 6, which is the working model of the concept.

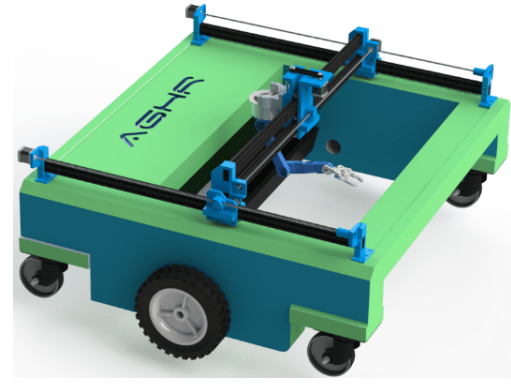

Figure 5. Harvesting robot

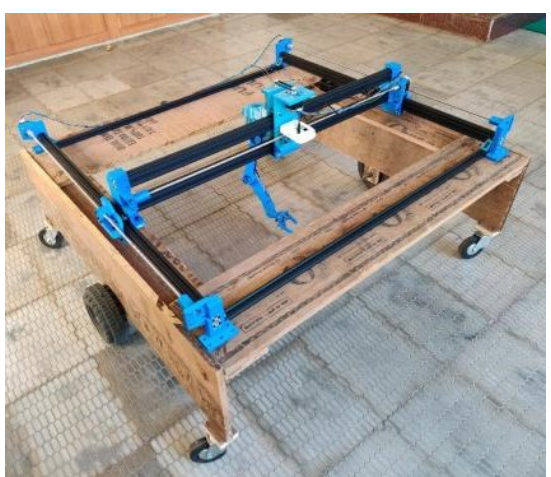

Figure 6. Final harvesting robot 


\section{RESULTS AND DISCUSSION}

The harvesting robot was tested in carrot and cantaloupe farming fields and gave us the following outputs, as shown in Table 1. The vision system was performed using the YOLOV3 algorithm. The robot was able to detect 232 out of 300 carrots with an average accuracy of 93\% in 4 seconds. Similarly, 245 out of 300 cantaloupes were detected, leading to an average accuracy of $95 \%$ in 2 seconds. It was also able to differentiate between the fully grown crops and the growing ones by feeding the neural network dataset with more images.

Table 1. The output of accuracy and the time is taken for each crop

\begin{tabular}{ccccc}
\hline No. & Type of crop & $\begin{array}{c}\text { No. of crops } \\
\text { detected }\end{array}$ & $\begin{array}{c}\text { Average accuracy } \\
(\%)\end{array}$ & $\begin{array}{c}\text { Time taken to complete the detection } \\
\text { (seconds) }\end{array}$ \\
\hline 1 & Carrot & 232 out of 300 & 93 & 4 \\
2 & Cantaloupe & 245 out of 300 & 95 & 2 \\
\hline
\end{tabular}

As the YOLOV3 algorithm also enables us to detect multiple classes, it can be used in multiple fields which contain different crops. The proposed robotic manipulator was able to acquire the crop in approximately 18 seconds. It is too high when compared to the time taken by a manual process, but still, as humans grow tiresome, this result can be acceptable due to the deviations in the robot navigation paths caused by the farming field's obstacles such as dirt and stones. The waypoint navigation by using a global position system gave an accuracy of around $87 \%$.

\section{CONCLUSION}

An integrated robot system was designed, and the system was tested against several products using software in order to check its validity. A prototype of the designed system was implemented and verified in the form of hardware. In this development, automatic crop harvesting was performed through the method of position detection and harvesting using a robotic manipulator with a harvesting hand that does not damage the crop. The accuracy of the detection of crops such as carrots and cantaloupe was found, and the time taken for the detection of crops was verified. This robot may not replace a human but can work collaboratively as its performance doesn't exceed the levels of a human. This collaborative robot can be used in small-medium scale farms, which do not involve complete automation, yet the production rate increases. In the distant future, as unskilled labor gets eradicated, this harvesting bot will play a crucial role in fixing that problem. The current agricultural industry is going through hardship, and this product is set to make a breakthrough when it reaches the market as it is affordable and simple to use, which the farmers expect.

\section{ACKNOWLEDGEMENTS}

The author would like to thank the Department of Robotics and Automation of PSG College of Technology for supporting this project.

\section{REFERENCES}

[1] A. V. V. Koundinya and P. P. Kumar, "Indian vegetable seeds industry: status and challenges," International Journal of plant, animal and environmental sciences, vol.4, no.4, pp. 62-69, 2014.

[2] N. P. T. Anh, S. Hoang, D. Van Tai, and B. L. C. Quoc, "Developing Robotic System for Harvesting Pineapples," 2020 International Conference on Advanced Mechatronic Systems (ICAMechS), pp. 39-44, 2020, doi: 10.1109/ICAMechS49982.2020.9310079.

[3] W. Jia, Y. Zhang, J. Lian, Y. Zheng, D. Zhao, and C. Li, "Apple harvesting robot under information technology: A review," International Journal of Advanced Robotic Systems, vol. 17, no. 3, 2020, doi: 10.1177/1729881420925310.

[4] R. R. Shamshiri, C. Weltzien, I. A. Hameed, and I. J. Yule, "Research and development in agricultural robotics: A perspective of digital farming," International Journal of Agricultural and Biological Engineering, vol. 11, no. 4, pp. 1-11, 2018, doi: 10.25165/j.ijabe.20181104.4278

[5] G. Kootstra, X. Wang, P. M. Blok, J. Hemming, and E. V. Henten, "Selective Harvesting Robotics: Current Research, Trends, and Future Directions," Curr Robot Rep, vol. 2, pp. 95-104, 2021, doi: 10.1007/s43154-020-00034-1.

[6] A. Sembiring, A. Budiman, and Y. D. Lestari, "Design and Control of Agricultural Robot for Tomato Plants Treatment and Harvesting," Journal of Physics: Conference Series, vol. 930, 012019, 2017, doi: 10.1088/1742-6596/930/1/012019.

[7] A. D. Preter, J. Anthonis, and J. de Baerdemaeker, "Development of a Robot for Harvesting Strawberries," IFAC-PapersOnLine, vol. 51, no. 17, pp. 14-19, 2018, doi: 10.1016/j.ifacol.2018.08.054.

[8] D. Klaoudatos, V. Moulianitis, and N. Aspragathos, "Development of an Experimental Strawberry Harvesting Robotic System," Proceedings of the 16th International Conference on Informatics in Control, Automation and Robotics, 2019, doi: $10.5220 / 0007934004370445$. 
[9] M. U. Masood and M. A. Haghshenas-Jaryani, "Study on the Feasibility of Robotic Harvesting of Chile Pepper," Robotics 2021, vol. 10, pp. 94, doi: 10.3390/robotics10030094

[10] L. F. P. Oliveira, A. P. Moreira, and M. F. Silva, "Advances in Agriculture Robotics: A State-of-the-Art Review and Challenges Ahead," Robotics, vol. 10, no. 2, p. 52, 2021, doi: 10.3390/robotics10020052.

[11] Y. Sahu, R. K. Pateriya, and R. K. Gupta, "Cloud server optimization with load balancing and green computing techniques using dynamic compare and balance algorithm," 2013 5th International Conference and Computational Intelligence and Communication Networks. IEEE, 2013, doi: 10.1109/CICN.2013.114.

[12] L. Zhao and S. Li, “Object Detection Algorithm Based on Improved YOLOv3," Electronics, vol. 9, no. 3, p. 537, 2020, doi: 10.3390/electronics9030537.

[13] X. Wang, J. Liu, and X. Zhu, "Early real-time detection algorithm of tomato diseases and pests in the natural environment," Plant Methods, vol. 17, no. 1, 2021, doi: 10.1186/s13007-021-00745-2.

[14] M. O. Lawal, “Tomato detection based on modified YOLOv3 framework,” Sci Rep vol. 11, pp. 1447, 2021, doi: 10.1038/s41598021-81216-5.

[15] R. Yamashita, M. Nishio, R. K. G. Do, and K. Togashi, "Convolutional neural networks: an overview and application in radiology," Insights Imaging, vol. 9, pp. 611-629, 2018, doi: 10.1007/s13244-018-0639-9.

[16] L. Alzubaidi et al., "Review of deep learning: concepts, CNN architectures, challenges, applications, future directions," J. Big Data, vol. 8, pp. 53, 2021, doi: 10.1186/s40537-021-00444-8

[17] F. R. V. Alves and R. P. M. Vieira, "The Newton Fractal's Leonardo Sequence Study with the Google Colab," International Electronic Journal of Mathematics Education, vo. 15, no. 2, 2019, doi: 10.29333/iejme/6440.

[18] S. Srivastava, A. V. Divekar, C. Anilkumar, I. Naik, V. Kulkarni, and V. Pattabiraman, "Comparative analysis of deep learning image detection algorithms," J Big Data, vol. 8, pp. 66, 2021, doi: 10.1186/s40537-021-00434-w.

[19] A. Kuznetsova, T. Maleva, and V. Soloviev, "Using YOLOv3 Algorithm with Pre- and Post-Processing for Apple Detection in Fruit-Harvesting Robot," Agronomy, vol. 10, no. 7, p. 1016, 2021, doi: 10.3390/agronomy10071016.

[20] K. Osorio, A. Puerto, C. Pedraza, D. Jamaica, and L. Rodríguez, “A Deep Learning Approach for Weed Detection in Lettuce Crops Using Multispectral Images,” AgriEngineering, vol. 2, no. 3, pp. 471-488, 2020, doi: 10.3390/agriengineering2030032.

[21] G. Taylor, J. Li, D. Kidner, C. Brunsdon, and M. Ware, "Modelling and prediction of GPS availability with digital photogrammetry and LiDAR," International Journal of Geographical Information Science, vol. 21, no. 1, pp. 1-20, 2007, doi: $10.1080 / 13658810600816540$

[22] I. ÜNal and M. Topakci, "Design of a Remote-controlled and GPS-guided Autonomous Robot for Precision Farming," International Journal of Advanced Robotic Systems, vol. 12, no. 12, 2017, doi: 10.5772/62059.

[23] I. Amundson, J. Sallai, X. Koutsoukos, and A. Ledeczi, "Mobile Sensor Waypoint Navigation via RF-Based Angle of Arrival Localization," International Journal of Distributed Sensor Networks, vol. 8, no. 7, 2017, doi: 10.1155/2012/842107.

[24] K. Hou, H. Sun, Q. Jia, and Y. Zhang, "An Autonomous Positioning and Navigation System for Spherical Mobile Robot," Procedia Engineering, vol. 29, pp. 2556-2561, 2012, doi: 10.1016/j.proeng.2012.01.350.

[25] S. Panzieri, F. Pascucci, and G. Ulivi, "An outdoor navigation system using GPS and inertial platform," IEEE/ASME Transactions on Mechatronics, vol. 7, no. 2, pp. 134-142, June 2002, doi: 10.1109/TMECH.2002.1011250.

[26] M. Doyle and J. Newman, "Analysis of capacity-rate data for lithium batteries using simplified models of the discharge process," Journal of Applied Electrochemistry, vol. 27, no. 7, pp. 846-856, 1997.

[27] P. S. Sanchez and F. R. Cortes, "Cartesian Control for Robot Manipulators," Robot Manipulators Trends and Development, vol. 20, no. 5, pp. 289-294, 2010, doi: 10.5772/9186.

[28] H. Hanafusa, T. Yoshikawa, and Y. Nakamura, "Analysis and Control of Articulated Robot Arms with Redundancy," IFAC Proceedings, vol. 14, no. 2, pp. 1927-1932, 1981, doi: 10.1016/s1474-6670 (17)63754-6.

[29] R. Jain, M. N. Zafar, and J. C. Mohanta, "Modeling and Analysis of Articulated Robotic Arm for Material Handling Applications," IOP Conference Series: Materials Science and Engineering, pp. 691, 2019, doi: 10.1088/1757-899x/691/1/012010.

[30] S. Albawi, T. A. Mohammed, and S. Al-Zawi, "Understanding of a convolutional neural network," 2017 International Conference on Engineering and Technology (ICET), pp. 1-6, 2017, doi: 10.1109/ICEngTechnol.2017.8308186.

[31] J. Schmalz and G. Reinhart, "Automated Selection and Dimensioning of Gripper Systems," Procedia CIRP, vol. 23, pp. 212-216, 2014, doi: 10.1016/j.procir.2014.10.080.

[32] Z. Tang, J. Lu, Z. Wang, and G. Ma, "The development of a new variable stiffness soft gripper," International Journal of Advanced Robotic Systems, vol. 16, no. 5, 2019, doi: 10.1177/1729881419879824.

[33] R. Kolluru, K. P. Valavanis, and T. M. Hebert, "Modeling, analysis, and performance evaluation of a robotic gripper system for limp material handling," IEEE Transactions on Systems, Man, and Cybernetics, Part B (Cybernetics), vol. 28, no. 3, pp. 480-486, June 1998, doi: 10.1109/3477.678660.

\section{BIOGRAPHIES OF AUTHORS}

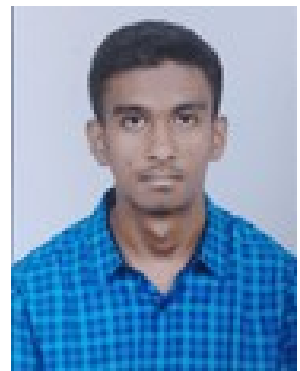

Vikram Raja (D) SC P is a B.E final year student pursuing his undergraduate program at PSG College of technology in the field of Robotics and Automation. He has done various projects which includes "Robot collaborative material handling system" which is an inventory management robot, IoT based water management system, 3 Axis laser CNC machine and currently working on the project titled "Quadruped robot" which is a legged mobile robot designed for achieving locomotion in rough terrain. His area of interest are Embedded systems, CNC machines, IOT based Home automation, Vision Systems, and Deep learning. He has also published a paper on the topic "Autonomous Indoor navigation for mobile robot". He can be contacted at email: vikramraja.ur@gmail.com. 


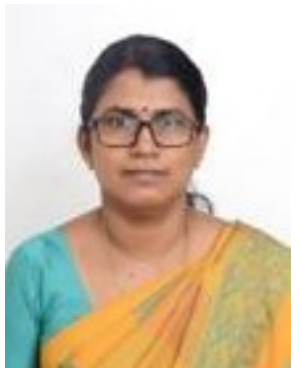

Bindu Bhaskaran (D) 88] SC P is currently working as Senior Grade Assistant Professor in the Department of Robotics and Automation Engineering, PSG College of Technology, Coimbatore, India. She completed her Bachelor's degree in Electrical and Electronics Engineering from Calicut University and Master's Degree in Energy Engineering from Anna University. She was awarded First Rank Gold Medal, Best Student and Best all-rounder in Master's Degree. She then obtained $\mathrm{PhD}$ in Electrical Engineering from Anna University Chennai in the year 2019. She has 16+ years of teaching experience and 2 years of industrial experience. Her areas of interest are Electrical machines, Control Systems, Power Electronics, Renewable Energy Systems and Electrical drives. She has published 8 papers in International Journals and authored one book in Basic Concepts of Smart Grid. She is a Life member of ISTE, IEI, IAENG, IAENG Society of Electrical Engineering, ISSE, and SESI. She can be contacted at email: bdu.rae@psgtech.ac.in.

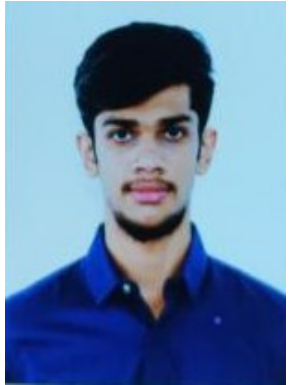

Koushik Karan Geetha Nagaraj (D) $8 \mathrm{SC}$ P is a B.E final year student pursuing his undergraduate program at PSG College of technology in the field of Robotics and Automation. He has completed projects which include Automated Thermostat Testing Station, Pick and Place Robot using robot operating system (ROS), and Robot Collaborative Material Handling System which is an inventory management robot. He has done his internship on automation system designs under Aatek Robo Private Limited. His area of interest is robot kinematics and dynamics, automation system designs, drones, mobile robots and medical robots. He is currently working on the project titled "Advancement on Robotic Endotrainer" which uses robot operating system (ROS) for simulation. He has published papers on the topic "Design of a Thermostat Testing Station", "Design and implementation of the 6-DoF robotic manipulator" and "Development of AI Chatbot to Learn Programming". He can be contacted at email: koushikkaran6@gmail.com.

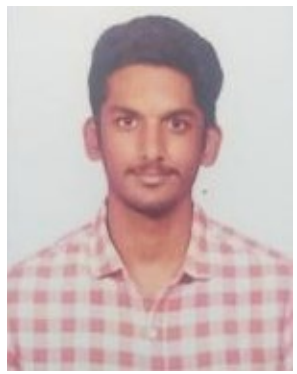

Jai Gowtham Sampathkumar (D) SC P is a B.E final year student pursuing his undergraduate program in PSG College of Technology in the field of Robotics and Automation. He completed his Diploma degree in Mechanical Engineering in PSG Polytechnic College. He was awarded as best project in Diploma degree. He has an implant training experience in PSG Industrial Institute during Diploma degree. He has hands on experience about 7 months on manufacturing processes, pattern making, and assembling industries. His area of interests are IoT and Embedded Systems, Mechanical Designing and modelling, Mobile Robots, CNC machines. He has done projects which includes Green Building, Self-Balancing Robot, and Green Building Automation. He is currently working on the project titled "Gesture Based Robot Arm Control". He can be contacted at email: jaigowtham.gow123@gmail.com.

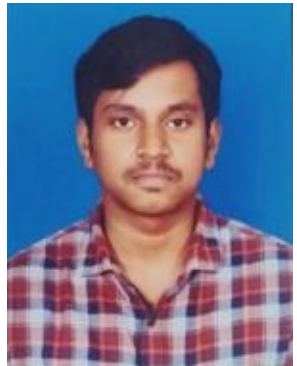

Shri Ram Senthilkumar (iD 8 SC $P$ is a B.E final year student pursuing his undergraduate program at PSG college of technology in the field of robotics and automation. His area of research focuses on Vision systems, NC machines, Internet of Things based home automation, Machine Learning, Embedded systems and Mobile Robots. He has done various projects which includes 'Green building Automation' which is based on Internet of things that would positively impact the future, 'Object tracking and following robot' that uses Computer vision and he is currently working on "Gesture Based Robot Arm Control". He can be contacted at email: shriramtg34@gmail.com. 